\title{
Diabetes as a risk factor in COVID - 19 Pandemic
}

\author{
Dr.Amit Bhushan Sharma \\ Consultant Physician \& Diabetologist \\ Apex Hospital, Jaipur, India \\ ABS Ployclinic Jaipur, India \\ Corresponding email: dramitbhushan@gmail.com
}

Background:Corona virus Disease-2019 popularly known as COVID-19 is a highly infectious disease caused by the novel corona virus. It spreads through close personal contact with someone who has the virus.

COVID-19 is caused by the corona virus SARS-CoV-2 (severe acute respiratory syndrome coronavirus-2), which has spread quickly to across the world.

- The spreading mechanism of the virus is primarily by transmission of respiratory droplets between people.

- The incubation time is on average 2-14days, followed by 1-2 weeks of symptomatic disease. It is characterized by a wide spectrum of symptoms including coughing, fever and respiratory problems such as viral pneumonia and respiratory failure.

- In worst cases, these can lead to death.

Keyword: corona virus, diabetics, infection

Diabetes as a risk factor in COVID - 19:

COVID-19 is a double challenge for people with diabetes. The corona virus may thrive in an environment of elevated blood glucose. Diabetes has been reported to be a risk factor for the severity of the disease. When people with diabetes develop a viral infection, it can be harder to treat due to fluctuations in blood glucose levels and the presence of diabetes complications.

People with high or unmanaged blood sugar levels have less than normal blood flow, which makes it difficult for the body to harness nutrients, natural defences meant to protect the body against several infections and promote healing. Hence, it usually takes a little longer than usual for people with diabetes to recover as well.

There appear to be two reasons for this. Firstly, the immune system is compromised, making it harder to fight the virus and likely leading to a longer recovery period. Secondly, the virus may thrive in an environment of elevated blood glucose. Diabetes patients have impaired immuneresponse to infection both in relation to cytokine profile and to changes in immuneresponses including $\mathrm{T}$-cell and macrophage activation. 
Poor glycaemic control impairs several aspects of the immune response to viral infection and also to the potential bacterial secondary infection in the lungs. It is likely that many of the patients with diabetes in world have been in poor metabolic control when infected by COVID19 infection.

As per the statistics of WHO, prevalence of diabetes in India in the year 2000 was 31,705,000 and will be around $79,441,000$ by 2020 . So the situation becomes more alarming in the countries like India.

When comparing intensive care and non-intensive care patients with COVID-19, there appears to be a twofold increase in the incidence of patients in intensive care having diabetes. Mortality seems to be about threefold higher in people with diabetes compared with the general mortality of COVID-19.

The number of co-morbidities is a predictor of mortality in COVID-19. In addition to diabetes, the other common comorbidities are hypertension, cardiovascular disease, and lung disease. Indeed, people with diabetes are a high-risk group for severe disease. Notably, diabetes was also a risk factor for severe disease and mortality in the previous SARS, MERS (Middle East respiratory syndrome) corona virus infections and the severe influenza AH1N1 pandemic in 2009.

Many patients with type 2 diabetes are obese and obesity is also a risk factor for severe infection.

It was analyzed during the influenza A H1N1 epidemic in 2009 that the disease was more severe and had a longer duration in about twofold more patients with obesity who were then treated in intensive care units compared with background population. Specially, metabolic active abdominal obesity is associated with higher risk.

The abnormal secretion of adipokines and cytokines like TNF-alfa and interferon characterize a chronic low-grade in abdominal obesity and may induce an impaired immune-response. People with severe abdominal obesity also have mechanical respiratory problems, with reduced ventilation of the basal lung sections increasing the risk of pneumonia as well as reduced oxygen saturation of blood. Obese subjects also have an increased asthma risk, and those patients with obesity and asthma have more symptoms, more frequent and severe exacerbations and 
reduced response to several asthma medications.

The most frequent co-morbidities to COVID 19 are hypertension and diabetes. Both diseases are often treated with angiotensin-converting enzymes (ACE) inhibitors. Corona virus binds to target cells through angiotensin-converting enzyme 2 (ACE2), which expressed in the epithelial cells in the lungs, blood vessels and in the intestine. In patients treated with ACE and angiotensin II receptor blockers, expression of ACE2 is increased. Therefore, it has been suggested that ACE2 expression may be increased in these two groups of patients with hypertension and diabetes, which could facilitate infection with COVID-19 and increase the risk of severe disease and fatality.

Lastly, late diabetic complications such as diabetic kidney disease and ischaemic heart disease may complicate the situation for people with diabetes, making them frailer and further increasing the severity of COVID-19 disease and the need for care such as acute dialysis. Some findings indicate that COVID-19 could cause acute cardiac injury with heart failure, leading to deterioration of circulation.

\section{Treatment of Diabetes during COVID 19} infection:

Poor glycaemic control is a risk factor for serious infections and adverse outcomes. However, the reverse is also true and the risk of infection, including bacterial pneumonia, can be reduced through good glycaemic control.

The problem is that infections cause loss of glycaemic control, and treatment of hyperglycaemia is difficult during inter current disease with fever, unstable food intake and use of drugs like glucocorticoids in patients with respiratory problems. To maintain optimal glycaemic control requires more frequent blood glucose monitoring and continuous change in antidiabetic treatment after the measured glucose levels.

In patients with type 2 diabetes, metformin and SGLT-2 inhibitors with moderate to severe illness should be stopped. Dipeptidyl peptidase 4 (DPP-4) inhibitors and also linagliptin can be used in patients with impaired kidney function without risk of hypoglycaemia. Sulphonylureas may induce hypoglycaemia in patients with low calorie intake.

In many patients with type 2 diabetes, insulin treatment will be preferred and need to be initiated, which is 
complicated because of the limited time for instruction and titration of insulin. Patients already treated with basal insulin will need fast-acting bolus insulin to correct hyperglycaemia. Hospitals have experience and algorithms for the treatment of patients during intercurrent disease, but the time involved for treating labile glycaemic control is a major problem in situations where time is short.

In patients with type 1 diabetes treated with basal bolus or insulin pump therapy, the insulin doses should be titrated using frequent glucose and ketone monitoring to avoid hypoglycaemia in patients with reduced food intake, and adding correctional bolus of fast-acting insulin to avoid severe hyperglycaemia and ketoacidosis.

Taken all together, patients with diabetes are a high-risk and complicated group of patients to treat for COVID-19, with an increased requirement of hospitalization. Patients with diabetes need intensive attention to reduce the risk of fatalities. Patients with diabetes should follow the general prevention advice given by the authorities thoroughly to avoid infection with COVID-19.

\section{References:}

1. Del Rio C, Malani PN. COVID-19- new insights on a rapidly changing epidemic . JAMA. 2020

2. Huttunen R, Syrjänen J. Obesity and the risk and outcome of infection. Int $\mathrm{J}$ Obes (Lond). 2013;37: 333-40.

3. Hoffmann M, Kleine-Weber H, Schroeder $\mathrm{S}$, et al. SARS-CoV-2 cell entry depends on ACE2 and TMPRSS2 and is blocked by a clinically proven protease inhibitor.

4. www.who.int $>$ diseases

5. https://mohfw.gov.in 